\title{
Modélisation par la méthode Bond Graph d'un système combiné plancher chauffant-plafond rafraîchissant
}

\author{
Abdelatif Merabtine ${ }^{1, a}$, Salim Mokraoui $^{1}$, Riad Benelmir $^{1}$ et Najib Laraqi ${ }^{2}$ \\ 1 Université de Lorraine, LERMAB, Faculté des sciences et Technologie, 54506 Vandoeuvre-Lès-Nancy, France \\ 2 Université Paris Ouest, LTIE, EA 4415, GTE, 50 rue de Sèvres, 92410 Ville d'Avray, France
}

Reçu le 24 août 2011, accepté le 13 décembre 2011

\begin{abstract}
Résumé - Aujourd'hui, différents types de systèmes de Chauffage, de Ventilation et de Conditionnement de l'air (CVC) sont utilisés. À un niveau équivalent de confort thermique, la consommation énergétique diffère de l'un à l'autre. De ce fait, il est plus raisonnable de choisir le système garantissant un meilleur rapport confort thermique/consommation énergétique. Dans cette étude, un système combiné plancher chauffantplafond rafraîchissant a été considéré. Afin d'estimer la fiabilité de ce type de systèmes CVC, le comportement thermique d'un bâtiment simple intégrant cette technique de chauffage et de rafraîchissement a été modélisé. Le modèle développé, utilisant l'approche Bond Graph (BG), a servi d'outil de simulation afin d'évaluer les performances énergétiques du système combiné. Il s'est avéré que cette technique répond aux besoins en chaud et en froid tout en maintenant un meilleur confort thermique. Cependant, elle est bien adaptée dans les climats relativement secs et froids.
\end{abstract}

Mots clés : CVC / plancher chauffant / plafond rafraîchissant / Bond Graph / comportement thermique du bâtiment

\begin{abstract}
Dynamic model of the floor heating system combined with the roof cooling system using the Bond Graph approach. Heating, Ventilation and Air-Conditioning (HVAC) systems are commonly used in both residential and commercial buildings. Although many of these systems can provide the necessary thermal comfort level, they often lead to excessive energy consumption. Hence, reliable HVAC systems are required in order to comply with environmental constraint while providing the best thermal comfort- energy consumption ratio. In this paper, thermal behavior of a simplified building comprised of two similar adjacent spaces has been modeled in order to assess the performance of a combined floor radiant heating and ceiling radiant cooling. The thermal simulation of the building has been performed by using the Bond Graph modeling approach, where both temperatures and heat flux annual profiles have been calculated. Comparison with TRNSYS software results has also been made. It was found that the combined floor radiant heating and ceiling radiant cooling is able to meet energy demand of the building insuring a good thermal comfort level. However, the selected heating/cooling technique is rather more useful for cold and dry climate.
\end{abstract}

Key words: HVAC / floor radiant heating / ceiling radiant cooling / Bond Graph / thermal behavior of building

\section{Introduction}

Le plancher chauffant (Fig. 1) suscite un regain d'intérêt pour son efficacité énergétique et le confort thermique de l'habitat qu'il procure (uniformité de la température surfacique et aussi faible valeur de la température opérative) [1]. L'idée consiste à porter la

\footnotetext{
a Auteur pour correspondance :

abdelatif.merabtine@lermab.uhp-nancy.fr
}

surface intérieure du plancher à une température maximale de $27{ }^{\circ} \mathrm{C}$. Le transfert de chaleur rayonnement est significatif. Par conséquent, cette technique procure un confort thermique supérieur aux autres types de chauffage car la chaleur est répartie uniformément.

Il paraît donc judicieux d'utiliser le même type de système en rafraîchissement. Dans ce cas, c'est de l'eau glacée à environ $15{ }^{\circ} \mathrm{C}$ qui circule dans les tubes au-dessus du faux plafond (Fig. 2). 


\section{Nomenclature}

\begin{tabular}{|c|c|c|}
\hline$A$ & Surface & $\mathrm{m}^{2}$ \\
\hline$C$ & Capacité thermique & J.K $\mathrm{K}^{-1}$ \\
\hline$c_{\mathrm{P}}$ & Chaleur spécifique à pression constante (extérieure du tube) & J.kg ${ }^{-1} \cdot \mathrm{K}^{-1}$ \\
\hline$c_{\mathrm{V}}$ & Chaleur spécifique à volume constante & $\mathrm{J} . \mathrm{kg}^{-1} \cdot \mathrm{K}^{-1}$ \\
\hline$D$ & Diamètre du tube & $\mathrm{m}$ \\
\hline$d$ & Diamètre intérieur & $\mathrm{m}$ \\
\hline$E$ & Épaisseur & $\mathrm{m}$ \\
\hline$h$ & Enthalpie spécifique & ${\mathrm{J} . \mathrm{kg}^{-1}}^{-1}$ \\
\hline$H$ & Enthalpie totale & $\mathrm{J}$ \\
\hline$\dot{H}$ & Flux d'enthalpie & J.s ${ }^{-1}$ \\
\hline$m$ & Masse du fluide & $\mathrm{kg}$ \\
\hline$\dot{m}$ & Débit massique & kg.s ${ }^{-1}$ \\
\hline$N u$ & Nombre de Nusselt & - \\
\hline$P$ & Pression absolue & $\mathrm{Pa}$ \\
\hline$Q$ & Chaleur totale & $\mathrm{J}$ \\
\hline$\dot{Q}$ & Flux de chaleur & $\mathrm{J} . \mathrm{s}^{-1}$ \\
\hline$R$ & Résistance thermique & $\mathrm{K} . \mathrm{W}^{-1}$ \\
\hline$R e$ & Nombre de Reynolds & - \\
\hline$T$ & Température absolue & K \\
\hline$U$ & Énergie interne & $\mathrm{J}$ \\
\hline$V$ & Volume du fluide & $\mathrm{m}^{3}$ \\
\hline$v$ & Vitesse du fluide & $\mathrm{m} \cdot \mathrm{s}^{-1}$ \\
\hline$\dot{V}$ & Débit volumique & $\mathrm{m}^{3} \cdot \mathrm{s}^{-1}$ \\
\hline \multicolumn{3}{|c|}{ Symboles grecs } \\
\hline$\alpha$ & Diffusivité thermique & $\mathrm{m}^{2} \cdot \mathrm{s}^{-1}$ \\
\hline$\lambda$ & Conductivité thermique & $\mathrm{W} \cdot \mathrm{m}^{-1} \cdot \mathrm{K}^{-1}$ \\
\hline$\nu$ & Viscosité cinématique & $\mathrm{m}^{2} \cdot \mathrm{s}^{-1}$ \\
\hline$\rho$ & Masse volumique & $\mathrm{kg} \cdot \mathrm{m}^{-3}$ \\
\hline \multicolumn{3}{|c|}{ Indices et exposants } \\
\hline 0 & Initiale & \\
\hline 1 & Zone 1 & \\
\hline 2 & Zone 2 & \\
\hline $\mathrm{C}$ & Chape béton & \\
\hline cond & conduction & \\
\hline conv & Convection & \\
\hline $\mathrm{D}$ & Basé sur le diamètre & \\
\hline e & Sortie (exit) & \\
\hline ext & Extérieur & \\
\hline $\mathrm{f}$ & Plancher (floor) & \\
\hline $\mathrm{g}$ & Sol (ground) & \\
\hline i & Entrée (inlet) & \\
\hline $\mathrm{P}$ & Caissons (plates) & \\
\hline ray & Rayonnement & \\
\hline $\mathrm{S}$ & Dalle sur terre-plein (Slab) & \\
\hline $\mathrm{Spc}$ & $\begin{array}{l}\text { Température de consigne pendant } \\
\text { une période hivernale (Set-point for cold days) }\end{array}$ & \\
\hline Sph & $\begin{array}{l}\text { Température de consigne pendant } \\
\text { une période estivale (Set-point for hot days) }\end{array}$ & \\
\hline
\end{tabular}

Un système intégrant un plancher chauffant et un plafond rafraîchissant pourrait être une solution pour obtenir un meilleur compromis confort thermique/consommation.

Différents travaux ont été menés sur les systèmes utilisant de telles techniques : seules ou combinées $[1,2]$. D'autres auteurs ont été intéressés par le plancher intermittent fonctionnant en mode de chauffage en hiver et rafraîchissement en été $[3,4]$. Certains ont étudié la possibilité d'intégrer seulement des plafonds rafraîchissants dans les bâtiments tertiaires [5], le chauffage étant assuré par des convecteurs. Les systèmes combinés ont été également étudiés, notamment ceux qui intègrent à la fois un système de plafond rafraîchissant et un système de ventilation [6] ou encore en ajoutant un déshumidificateur dans le cas des climats tropicaux [7]. Ce système a permis de réduire la consommation de $8 \%$ en énergie primaire. Cependant, il existe peu de travaux traitant le couplage plancher chauffant-plafond rafraîchissant. 


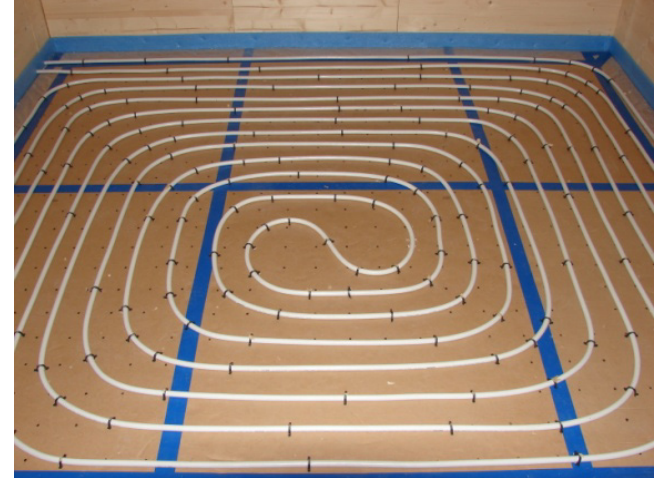

Fig. 1. Serpentin en polyéthylène constituant le plancher chauffant.

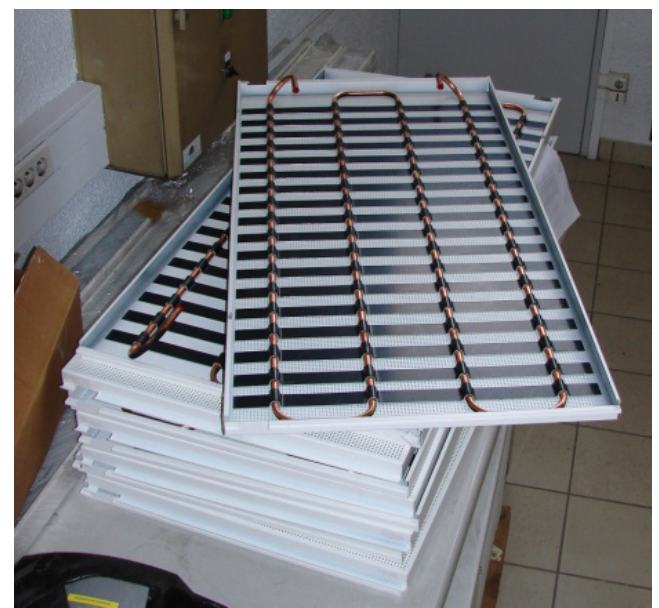

Fig. 2. Modules parcourus par une canalisation en cuivre constituant le plafond rafraîchissant.

Cet article présente donc une étude de modélisation d'un système combiné plancher chauffant-plafond rafraîchissant afin d'améliorer le rapport confort thermique/consommation énergétique. Une nouvelle approche de modélisation est proposée. Les Bond Graphs font partie de la catégorie de modèles dite de «boîte grise ». L'intérêt d'une telle approche, par rapport aux autres outils dédiés à la modélisation de la thermique du bâtiment comme TRNSys, est que ces derniers nécessitent l'écriture de nouveaux codes de calcul pour modéliser des composants qui ne sont pas disponibles dans leurs librairies, alors que les Bond Graphs, de part leur approche graphique et avec un minimum de paramètres d'entrée, nous épargnent cela. De plus ils sont très adaptés à la modélisation des problèmes élémentaires du transfert de chaleur 1D à travers une paroi tandis que TRNSys par exemple n'est pas destiné à ce type de problèmes.

\section{Présentation de la méthodologie Bond Graph}

Les Bond Graphs (graphes à lien) ont été imaginés par Paynter en 1961 [8]. Paynter est parti du concept que toute interaction entre systèmes physiques peut être représentée graphiquement par des liens. Le Bond Graph est un langage graphique basé sur les échanges énergétiques dans les systèmes. L'interaction entre deux composants est modélisée par une demi-flèche. La puissance $P$ qui s'échange entre eux est le produit de deux « variables de puissance » dont l'une est de type « effort généralisé » $e$ et l'autre de type «flux généralisé » $f$ [9] Les éléments BG utilisés dans la modélisation sont au nombre de neuf. Les éléments à un port représentent : l'élément d'inertie $(I)$, l'élément capacitif $(C)$, l'élément résistif $(R)$, la source d'effort $\left(S_{\mathrm{e}}\right)$ et la source de flux $\left(S_{\mathrm{f}}\right)$; les éléments à deux ports sont : l'élément transformateur $(T F)$ et l'élément gyrateur $(G Y)$. Les éléments à $n$ ports représentent les jonctions 1 et 0 permettant de connecter les éléments $I, C, R, S_{\mathrm{e}}$ et $S_{\mathrm{f}}$. La jonction 0 conserve le même effort tandis que la jonction 1 conserve le même flux [10].

Le choix des variables de puissance dépend du système physique à étudier. En thermique, l'effort est la température $T$ et la variable de flux est le flux d'entropie $\dot{S}$. Cependant, comme il est difficile de mesurer le flux d'entropie, les chercheurs ont introduit une pseudovariable de flux qui est le flux de chaleur $\dot{Q}$ [11]. Bien que le produit de $T$ et $\dot{Q}$ ne représente pas une puissance $[12,13]$, l'avantage est de rendre la modélisation des systèmes plus pratique.

\section{Description du modèle BG du circuit hydraulique}

Le modèle dynamique développé dans cette investigation considère le transfert de chaleur entre l'espace intérieur du bâtiment et l'environnement extérieur. Le couplage thermique entre l'enveloppe, le plancher chauffant et le plafond rafraîchissant a aussi été considéré. Le modèle permet d'avoir une estimation de la température moyenne de l'air intérieur, des températures surfaciques des parois, de la chaleur fournie par le plancher chauffant et absorbée par le plafond rafraîchissant, ainsi que les besoins annuels en chauffage et en refroidissement. La figure 3 présente le modèle BG « à mots » de l'ensemble du bâtiment étudié (Sect. 4). Bien que d'autres modèles BG, représentant les composants de la configuration étudiée dans la Section 4, sont interconnectés avec le modèle BG du système combiné, nous nous focalisons seulement sur la démarche de modélisation de ce dernier.

On considère les éléments constitutifs du plancher chauffant et du plafond rafraîchissant de la figure 4. Les frontières sont supposées adiabatiques. Dans le cas du plancher chauffant (Fig. 3a), le transfert de chaleur est convectif entre l'eau chaude et le tube. La chaleur fournie est ensuite partagée. Une partie est dissipée vers le sol par conduction $Q_{\mathrm{S}}$. L'autre partie est diffusée vers la chape béton $Q_{\mathrm{B}}$. Cette dernière est transférée à la zone intérieure et aux parois adjacentes par les phénomènes de convection et de rayonnement. Quant au plafond rafraîchissant (Fig. 3b), le transfert de chaleur est conductif, convectif et radiatif de part et d'autre le plafond. 


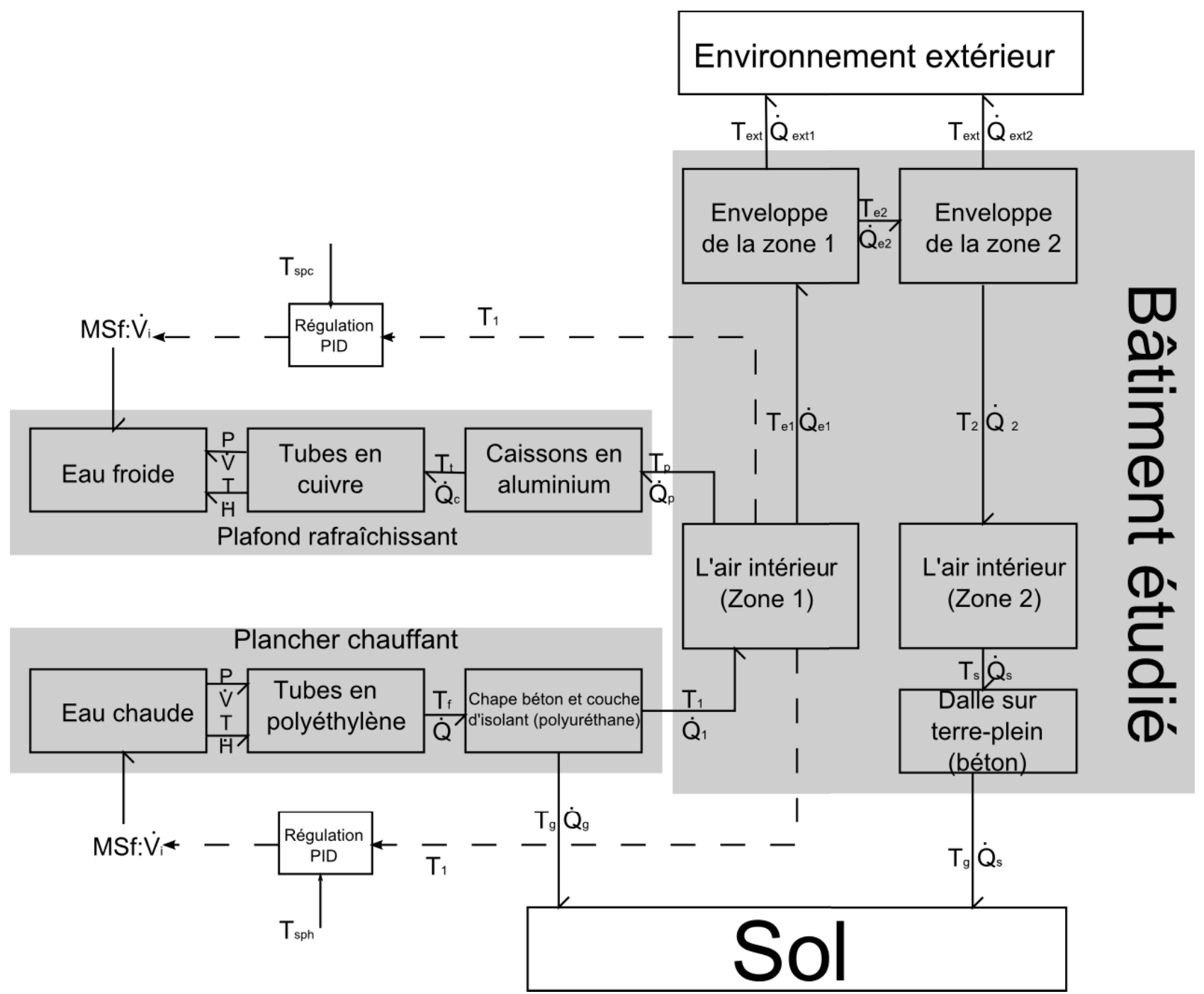

Fig. 3. Modèle BG à mots du bâtiment étudié (voir Sect. 4).

Le modèle BG développé prend en considération l'interaction physique entre deux types d'énergie : l'énergie hydraulique, décrivant la circulation du fluide, et l'énergie thermique, décrivant la variation de l'énergie interne et la détermination de la température du fluide. Cette interaction est modélisée, comme nous allons voir, par l'élément capacitif thermohydraulique $C$ et l'élément résistif $R$ à 2-ports.

Dans le cas d'un fluide incompressible, nous avons choisi les deux variables de déplacement généralisé suivantes : l'énergie interne $U$ et le volume $V$. À ces variables de déplacement on fait correspondre, pour l'énergie thermique, la température $T$ et le flux d'enthalpie $\dot{H}$ et, pour l'énergie hydraulique, la pression $P$ et le débit volumique $\dot{V}$. La détermination de l'énergie interne $U$ est faite dans l'élément $C$ représentant l'énergie stockée par le fluide, tandis que l'élément $R$ à 2-ports est utilisé pour calculer le débit volumique $\dot{V}$ et le flux d'enthalpie $\dot{H}$. Cet élément représente la dissipation de l'énergie le long du circuit hydraulique.
On considère le volume de contrôle de la figure 5 . L'écoulement de l'eau ayant une pression $P$, une température $T$, un volume $V$, une masse $m$ et une énergie interne $U$, est supposé en régime établi [14]. Le transfert de chaleur au sein du volume de contrôle, est représenté par l'interconnection de l'élément $C$, correspondant à la capacité thermique de l'eau, de l'élément $R$ à 2-ports, traduisant la dissipation de l'énergie thermique à l'entrée et à la sortie du volume de contrôle [15], et de l'élément $R$ à 1-port, indiquant la diffusion de la chaleur par conduction à travers le circuit hydraulique vers la chape béton.

La figure 6 montre le modèle BG du circuit hydraulique. À travers les liens en continu représentant l'énergie hydraulique, nous déduisons les équations (1)(2). L'équation (3) est déduite à partir de l'énergie thermique (liens en pointillés). Étant donné que le fluide est incompressible, on obtient :

$$
\begin{aligned}
P_{i} & =P_{e} \\
\dot{V}_{i} & =\dot{V}_{e}
\end{aligned}
$$




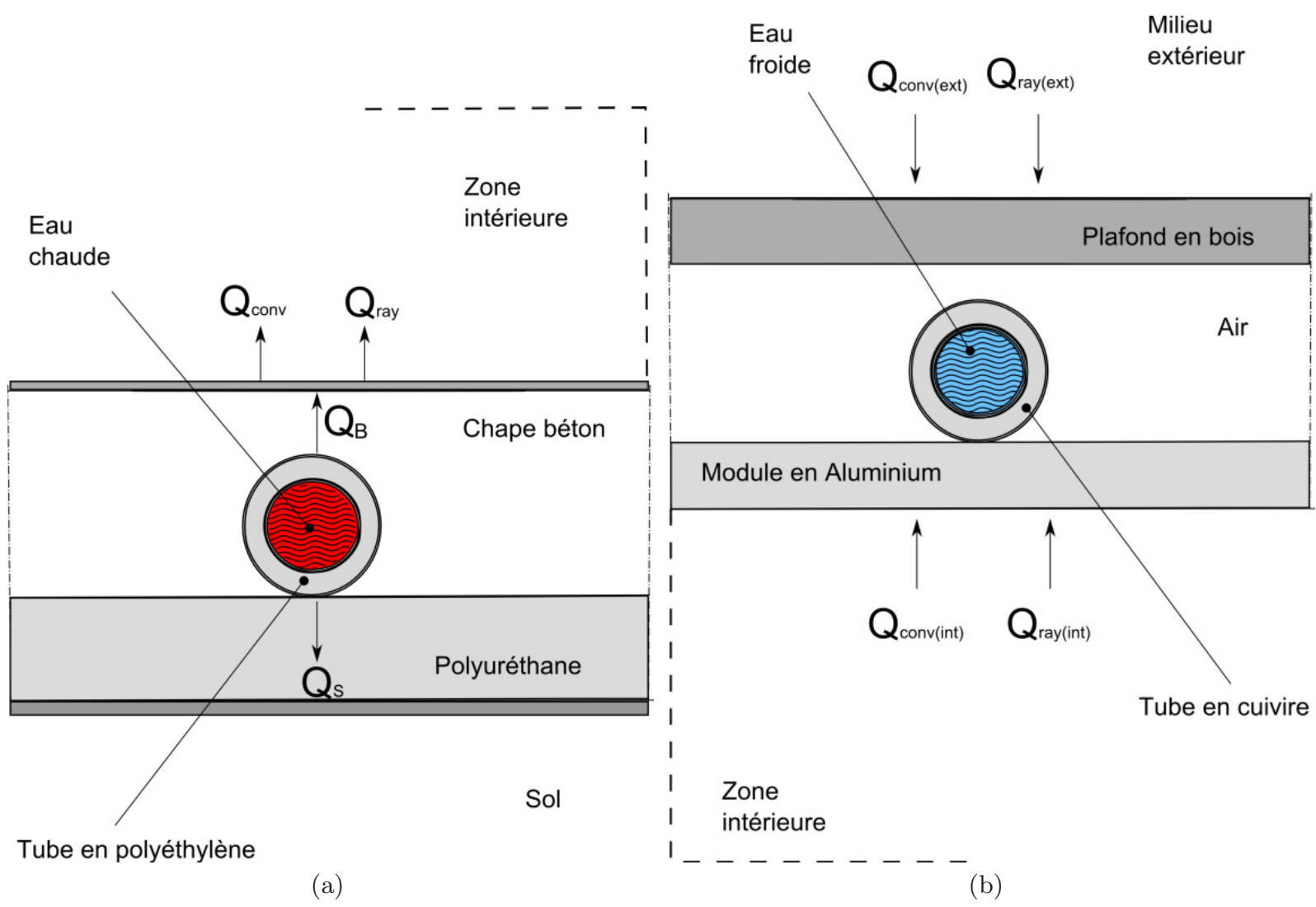

Fig. 4. Schémas des éléments constitutifs : (a) plancher chauffant; (b) plafond rafraîchissant.

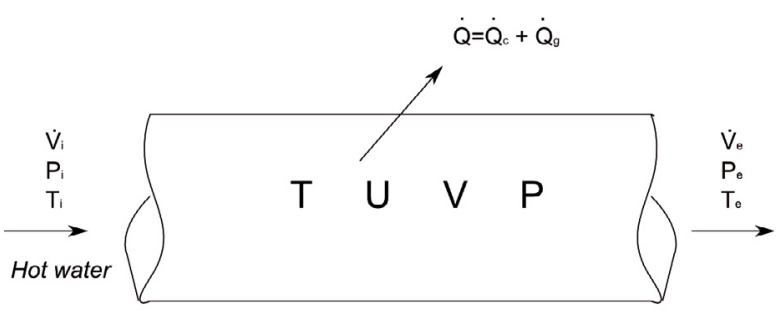

Fig. 5. Volume de contrôle.

Le bilan énergétique dans le volume, déduit à partir du modèle BG, s'écrit :

$$
\frac{\mathrm{d} U}{\mathrm{~d} t}+\dot{H}_{e}=\dot{H}_{i}+\dot{Q} \Rightarrow \frac{\mathrm{d} U}{\mathrm{~d} t}=\dot{H}_{i}-\dot{H}_{e}+\dot{Q}
$$

avec :

$$
\begin{aligned}
\dot{H}_{i} & =\rho \dot{V}_{i} c_{P} T_{i} \\
\dot{H}_{e} & =\rho \dot{V}_{e} c_{P} T_{e} \\
\dot{Q} & =\dot{Q}_{c}+\dot{Q}_{g}=\frac{T_{f}-T}{R A}
\end{aligned}
$$

$R$ représente la résistance thermique due au transfert convectif de la chaleur en provenance de l'eau chaude vers la surface intérieure du tube. $T_{\mathrm{f}}$ est la température de la surface externe des tubes à mi-hauteur du plancher.
Il est à noter que les équations (2) et (3) concordent bien avec les équations de la conservation de la masse et de l'énergie totale, respectivement. La satisfaction du bilan énergétique requiert une jonction 0 , à 3-ports, liée aux éléments $R$ à 2-ports traduisant la dissipation de l'énergie à l'entrée et à la sortie du volume de contrôle. Ils sont également connectés aux sources de températures aux frontières du volume de contrôle. Le couplage des énergies thermique et hydraulique se fait par le biais d'un lien « signal » fournissant la valeur du débit volumique nécessaire au calcul du flux d'enthalpie dans l'élément $R$ à 2-ports.

Par ailleurs, nous considérons un système réversible dans lequel il n'y a pas d'échange de travail à travers les frontières car $\mathrm{d} V \approx 0$. Ainsi nous obtenons :

$$
\mathrm{d} U=\rho V c_{V} \mathrm{~d} T
$$

L'équation (3) s'écrit donc sous cette forme :

$$
\begin{aligned}
\frac{\mathrm{d} U}{\mathrm{~d} T} & =\rho \dot{V}_{i} c_{P} T_{i}-\rho \dot{V}_{e} c_{P} T_{e}-\left[\frac{T-T_{f}}{R A}\right] \\
\frac{\mathrm{d} U}{\mathrm{~d} t} & =\rho \dot{V}_{i} c_{P} T_{i}-\rho \dot{V}_{e} c_{P} T_{e}-\frac{\left(U / \rho V c_{V}\right)}{R A}+\frac{T_{f}}{R A} \\
\frac{\mathrm{d} U}{\mathrm{~d} t} & =\rho \dot{V}_{i} c_{P} T_{i}-\rho \dot{V}_{e} c_{P} T_{e}+\frac{T_{f}}{R A}-U\left(\frac{1}{R A \rho V c_{V}}\right)
\end{aligned}
$$




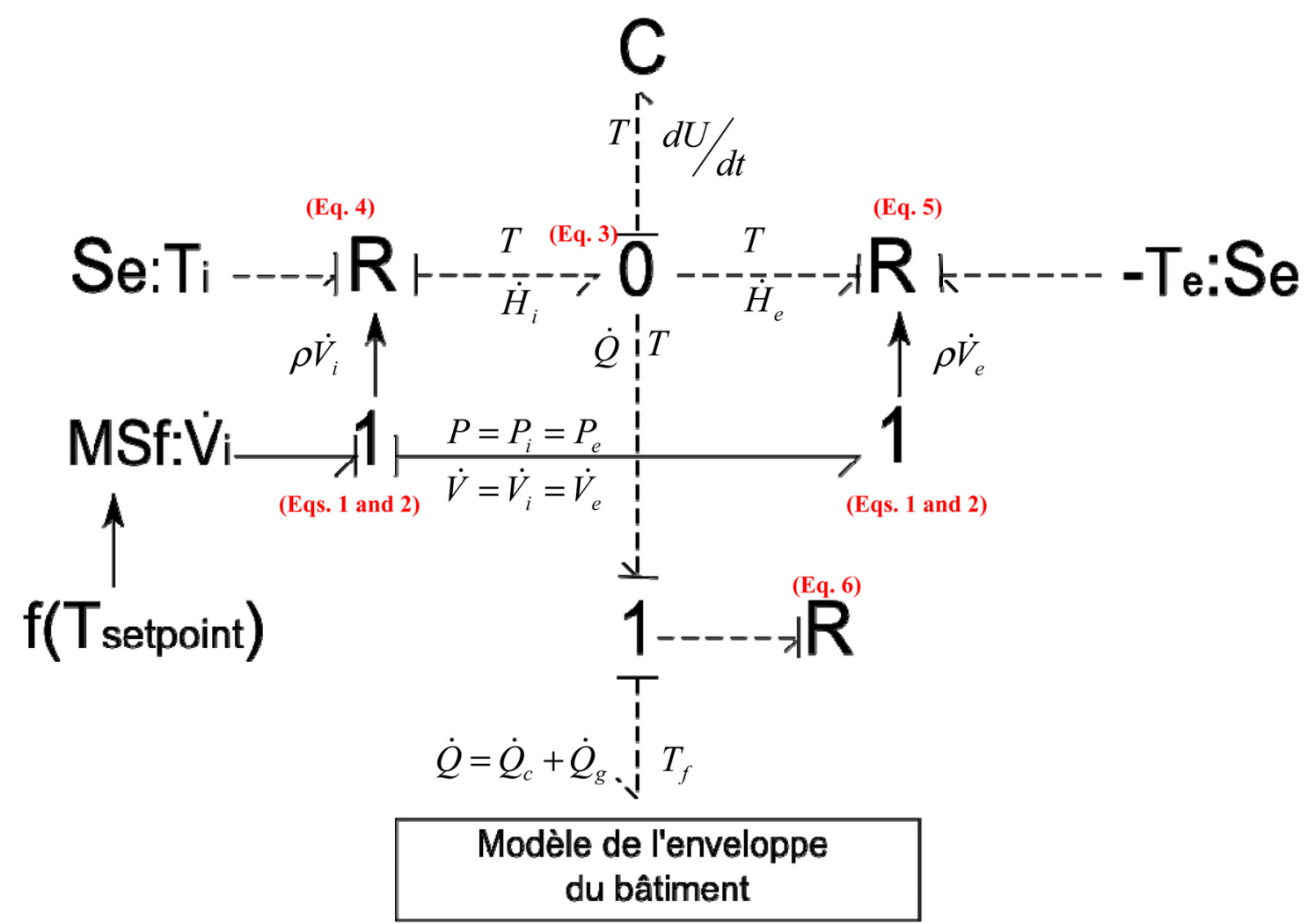

Fig. 6. Modèle - BG du circuit hydraulique (cas du plancher chauffant).

L'équation (8) est une équation différentielle de $1^{\text {er }}$ ordre. À l'issue de la solution de cette équation, nous obtenons la température $T$ du fluide par :

$$
T=\frac{U}{\rho V c_{V}}
$$

Le modèle BG de l'élément constructif du plancher chauffant est représenté dans la figure 7 . La capacité et la résistance thermique de chaque couche d'élément (ici le nombre de couches $n=4$ ) sont calculées par [16,17] :

$$
\begin{aligned}
C_{k} & =\left(\frac{m}{n+1}\right) c_{P} \\
R_{k} & =\frac{(e / n)}{\lambda A}
\end{aligned}
$$

La mise en équations du modèle de la figure 7 , permet de générer les équations (15)-(21) :

Les éléments $R: f_{k}=e_{k} / R_{k}$

$$
\begin{gathered}
R_{\text {global }}: Q_{1}=\frac{T_{f_{1}}-T_{1}}{R_{\text {global }}} \\
R_{f 1}: Q_{f 1}=\frac{T_{f}-T_{f 1}}{R_{f 1}} \\
R_{f 2}: Q_{f 2}=\frac{T_{f}-T_{f 2}}{R_{f 2}} \\
R_{\text {cond }}: Q_{g}=\frac{T_{f 2}-T_{g}}{R_{\text {cond }}}
\end{gathered}
$$

Les éléments $C: e_{k}=\frac{1}{C_{k}} \int f_{k} \cdot \mathrm{d} t$

$$
\begin{aligned}
C_{f 1}: \quad T_{f 1} & =\frac{1}{C_{f 1}} \int\left(\dot{Q}_{f 1}-\dot{Q}_{1}\right) \mathrm{d} t \\
C_{f}: T_{f} & =\frac{1}{C_{f}} \int\left(\dot{Q}_{f}-\dot{Q}_{f 1}-\dot{Q}_{f 2}\right) \mathrm{d} t \\
C_{f 2}: T_{f 2} & =\frac{1}{C_{f 2}} \int\left(\dot{Q}_{f 2}-\dot{Q}_{g}\right) \mathrm{d} t
\end{aligned}
$$

Dérivons les équations (16)-(18), nous obtenons le système d'équations différentielles suivant :

$$
\begin{aligned}
\frac{\mathrm{d} T_{f 1}}{\mathrm{~d} t} & =\frac{1}{C_{f 1}}\left[\frac{T_{f}-T_{f 1}}{R_{f 1}}-\frac{T_{f 1}-T_{1}}{R_{\text {global }}}\right] \\
\frac{\mathrm{d} T_{f}}{\mathrm{~d} t} & =\frac{1}{C_{f}}\left[\frac{T-T_{f}}{R}-\frac{T_{f}-T_{f 1}}{R_{f 1}}-\frac{T_{f}-T_{f 2}}{R_{f 2}}\right] \\
\frac{\mathrm{d} T_{f 2}}{\mathrm{~d} t} & =\frac{1}{C_{f 2}}\left[\frac{T_{f}-T_{f 2}}{R_{f 2}}-\frac{T_{f 2}-T_{g}}{R_{\text {cond }}}\right]
\end{aligned}
$$

Une fois le système résolu, la température de l'eau chaude circulant dans les tubes en polyéthylène est déterminée. La même démarche de modélisation a été utilisée dans le cas du plafond rafraîchissant, sauf que le transfert de chaleur se fait par conduction (à travers le caisson d'aluminium), par convection (à travers la lame d'air) et par rayonnement (à travers le plafond en bois). 


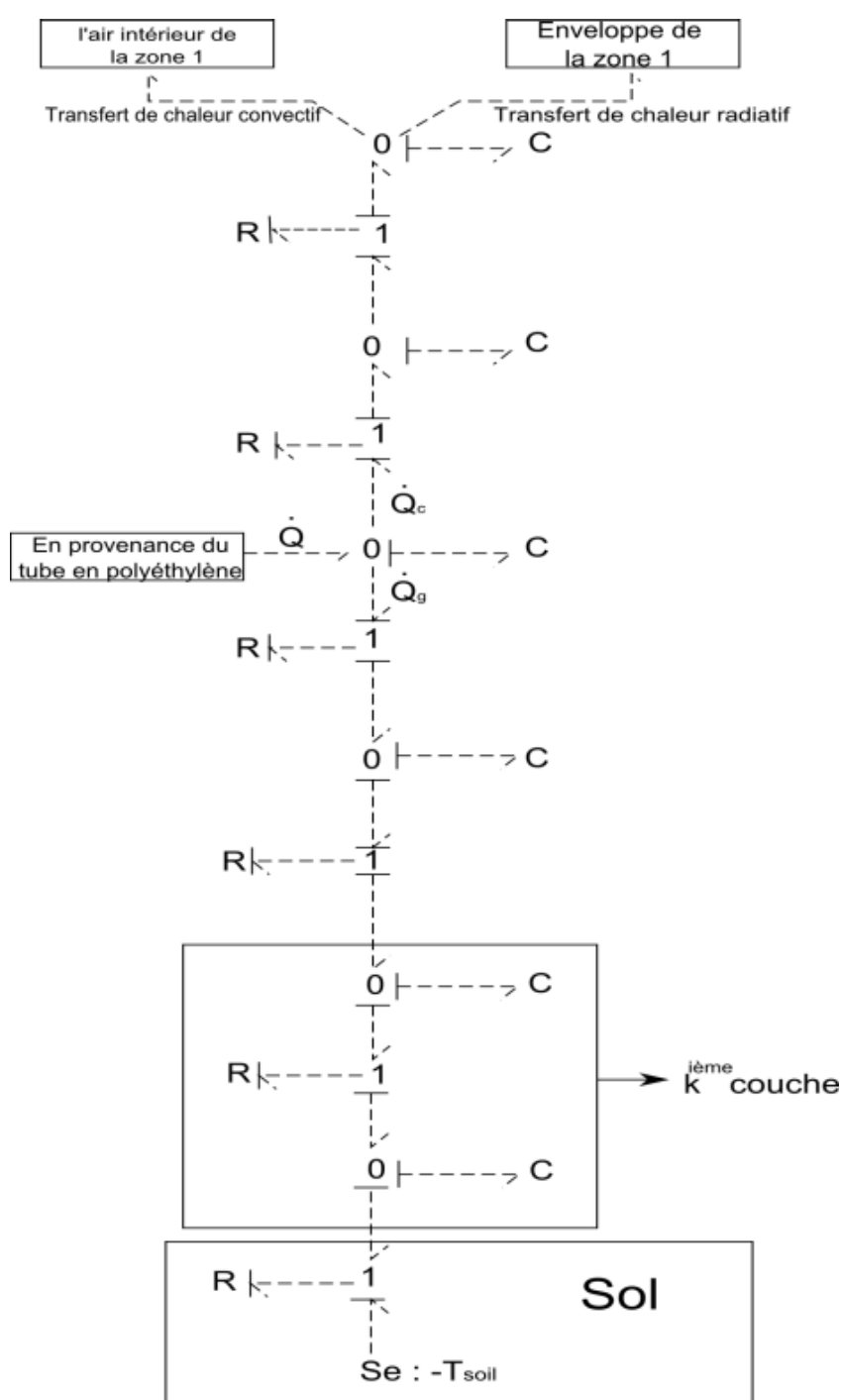

Fig. 7. Modèle Pseudo - BG de l'élément constructif du plancher chauffant.

\section{Modèle BG : Validation et simulations}

\subsection{Description du bâtiment étudié}

Le bâtiment étudié occupe une surface au sol approximative de $18 \mathrm{~m}^{2}$ pour une hauteur d'environ 2,3 m. La structure est divisée en deux chambres de dimensions identiques ( $9 \mathrm{~m}^{2}$ chacune). La zone 1 est équipée d'un plancher chauffant et d'un plafond refroidissant. Ce système combiné consiste à alimenter cette chambre en besoin chaud et en froid. Le tableau 1 donne les propriétés thermophysiques des matériaux constituant l'enveloppe ainsi que les caractéristiques des circuits hydrauliques. On cherchera ainsi à créer des conditions de température satisfaisant un meilleur confort thermique.

Les scénarii de fonctionnement du système sont présentés dans le tableau 2. La régulation de type PID assure, en fonction de la température de consigne, le changement des scénarii de fonctionnement. La zone 2 n'est pas régulée en température.
Les capacités thermiques des parois verticales, du plancher et de la toiture ainsi que les résistances thermiques de conduction sont déterminées par les relations (13) et (14).

Les résistances superficielles, intérieure $\left(R_{\mathrm{i}}\right)$ et extérieure $\left(R_{\mathrm{e}}\right)$, suivantes sont utilisées [18-20], en tenant compte des coefficients de convection et de rayonnement (linéarisés).

\section{Résultats et discussion}

Les résultats des simulations du modèle global sont confrontés à ceux générés par le logiciel TRNSys. Le fichier météorologique de la ville de Nancy (zone H1b) a été utilisé. L'objet de l'étude est de montrer l'efficacité énergétique du système combiné et son impact sur le confort thermique, à travers le suivi de l'évolution des températures (intérieures et surfaciques) des deux zones. En absence de ventilation, le confort thermique devrait satisfaire une température de l'air comprise entre $20{ }^{\circ} \mathrm{C}$ et $26^{\circ} \mathrm{C}$, une température radiante entre $19{ }^{\circ} \mathrm{C}$ et $27^{\circ} \mathrm{C}$ et une humidité relative entre $40 \%$ et $70 \%[18,21]$. Le risque de condensation est alors pris en compte.

La figure 8 présente les évolutions annuelles des températures des deux chambres. Les lignes en pointillés délimitent les durées des modes de fonctionnement des régulateurs en fonction de la température intérieure de la zone 1 (Fig. 8a). L'évolution de la température intérieure de la zone 2 est présentée sur la figure $8 \mathrm{~b}$. Les valeurs sont basses en période hivernale. Ceci s'explique par l'absence du chauffage dans cette période. On constate également des écarts absolus inférieurs à $1^{\circ} \mathrm{C}$ pour toute la période de simulation.

La température surfacique des parois influence la sensation du confort thermique à parts égales avec la température de l'air. La figure 9 présente l'évolution des températures surfaciques (considérées homogènes) du plancher chauffant et du plafond rafraîchissant. Globalement, un bon accord entre les résultats de simulation a été constaté. Les écarts absolus sont inférieurs à $1{ }^{\circ} \mathrm{C}$. La figure montre aussi que la température surfacique du plancher chauffant n'excède pas $29{ }^{\circ} \mathrm{C}$ en période de chauffe (Fig. 9a). Cependant, pour répondre à l'ensemble des critères du confort, la condensation dans la tuyauterie devra être réduite ou éliminée. Pour cela la température de rosée, ainsi que la température surfacique du faux-plafond, ont été présentées (Fig. 9b). Pour toute la période simulée, le problème de condensation ne se posera pas.

La figure 10 compare les flux de chaleur issus respectivement du plancher chauffant (Fig. 10a) et du plafond refroidissant (Fig. 10b). Une concordance entre les résultats BG-TRNSys est notée.

Les besoins mensuels du bâtiment étudié en chauffage sont présentés dans la figure 11. Le climat appliqué dans cette étude exige un chauffage permanent pendant toute l'année. La consommation énergétique évaluée pour toute l'année est estimée à environ $313 \mathrm{kWhep} / \mathrm{m}^{2} / \mathrm{an}$. 
Tableau 1. Propriétés thermo physiques des matériaux.

\begin{tabular}{|c|c|c|c|}
\hline Désignation & $\begin{array}{c}\text { Conductivité thermique } \\
\lambda\left(\mathrm{W} \cdot \mathrm{m}^{-1} \cdot \mathrm{K}^{-1}\right)\end{array}$ & $\begin{array}{c}\text { Chaleur spécifique } \\
c_{p}\left(\mathrm{~J} \mathrm{~kg}^{-1} . \mathrm{K}\right)\end{array}$ & $\begin{array}{l}\text { Masse volumique } \\
\rho\left(\mathrm{kg} \cdot \mathrm{m}^{-3}\right)\end{array}$ \\
\hline \multicolumn{4}{|c|}{ Parois verticales $(e=8,4 \mathrm{~cm})$} \\
\hline Bois* & 0,13 & 1600 & 490 \\
\hline \multicolumn{4}{|c|}{ Toit $(e=20 \mathrm{~cm})$} \\
\hline Bois* $(6,2 \mathrm{~cm})$ & 0,13 & 1600 & 490 \\
\hline \multirow{2}{*}{$\begin{array}{l}\text { Faux-plafond Seulement } \\
\text { dans la zone } 1\end{array}$} & 61 & 460 & 7860 \\
\hline & 24,3 & 1004 & 1,29 \\
\hline \multicolumn{4}{|c|}{ Plancher $(e=11,5 \mathrm{~cm})$} \\
\hline Chape béton $(e=6 \mathrm{~cm})$ & 1,4 & 880 & 2300 \\
\hline $\begin{array}{l}\text { Polyuréthane }(e=5,5 \mathrm{~cm}) \\
\text { seulement dans la zone } 1\end{array}$ & 0,03 & 837 & 35 \\
\hline \multicolumn{4}{|c|}{ Systèmes de chauffage et de rafraîchissement } \\
\hline \multicolumn{4}{|c|}{ Diamètres des tubes } \\
\hline Circuit chaud & \multicolumn{2}{|c|}{$\begin{array}{l}D=1,7 \mathrm{~cm} ; \mathrm{d}=1,5 \mathrm{~cm} \\
D=1.2 \mathrm{~cm} ; \mathrm{d}=1.0 \mathrm{~cm}\end{array}$} & $6290 \mathrm{~cm}$ \\
\hline Circuit froid & \multicolumn{2}{|c|}{$\begin{array}{l}\qquad D=1,2 \mathrm{~cm} ; \mathrm{d}=1,0 \mathrm{~cm} \\
\text { midité de } 10 \% \pm 3 \% .\end{array}$} & $4280 \mathrm{~cm}$ \\
\hline
\end{tabular}

Tableau 2. Scénarii de fonctionnement.

\begin{tabular}{ccc}
\hline Température $\left({ }^{\circ} \mathrm{C}\right)$ & Plancher chauffant & Plafond rafraîchissant \\
\hline$T<20$ & ON & OFF \\
$T>25$ & OFF & ON \\
$20 \leq T \leq 25$ & OFF & OFF
\end{tabular}

Température d'entrée de l'eau chaude $\left({ }^{\circ} \mathrm{C}\right) \quad$ Température d'entrée de l'eau glacée $\left({ }^{\circ} \mathrm{C}\right)$

Tableau 3. Résistances superficielles.

\begin{tabular}{|c|c|c|c|}
\hline \multicolumn{2}{|c|}{ Type de paroi } & $R_{\mathrm{i}}\left(\mathrm{m}^{2} \cdot \mathrm{K} \cdot \mathrm{W}^{-1}\right)$ & $R_{\mathrm{e}}\left(\mathrm{m}^{2} \cdot \mathrm{K} \cdot \mathrm{W}^{-1}\right)$ \\
\hline \multicolumn{2}{|c|}{ Paroi verticale } & 0,13 & 0,04 \\
\hline \multirow{2}{*}{ Paroi horizontale } & Plafond & 0,10 & 0,04 \\
\hline & Plancher & 0,17 & - \\
\hline
\end{tabular}

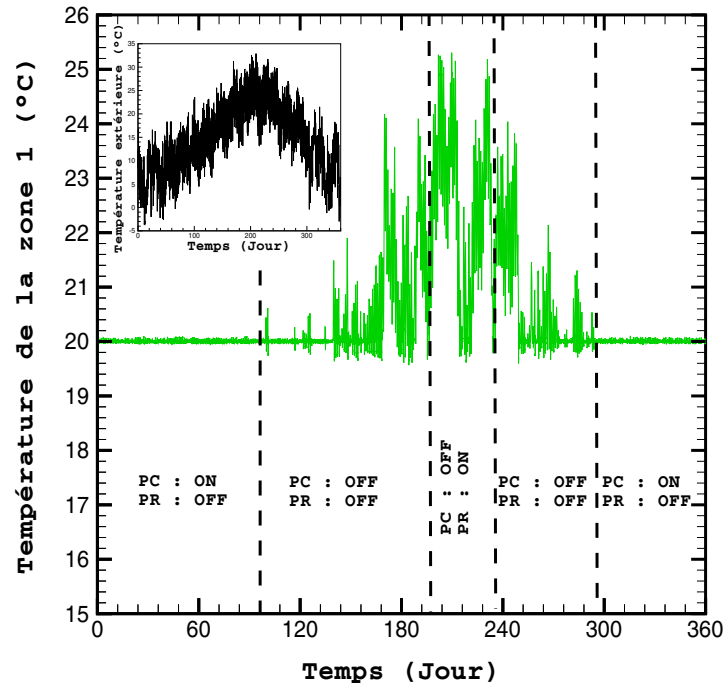

PC : Plancher Chauffant.
PR : Plafond Rafraîchissant.

(a)

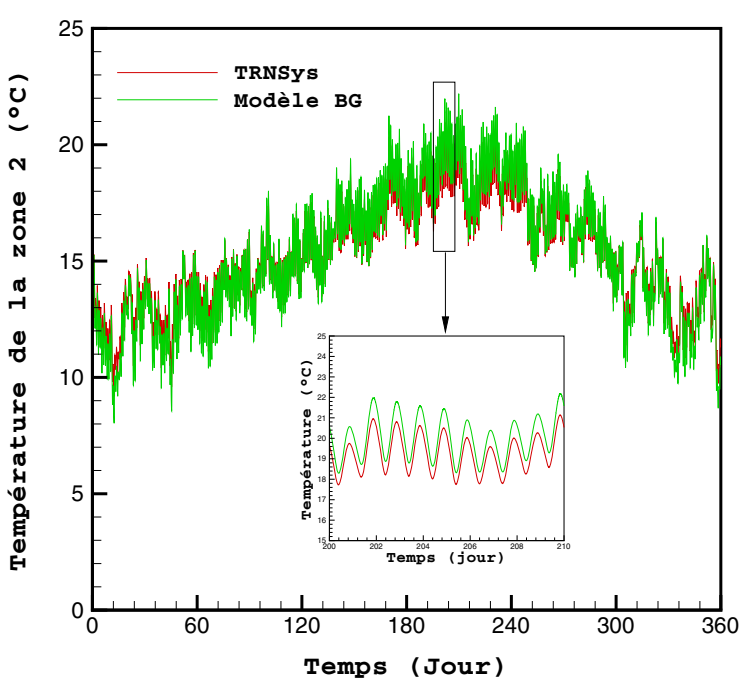

(b)

Fig. 8. Évolution de la température de l'air intérieur. (a) Zone 1; (b) zone 2. 


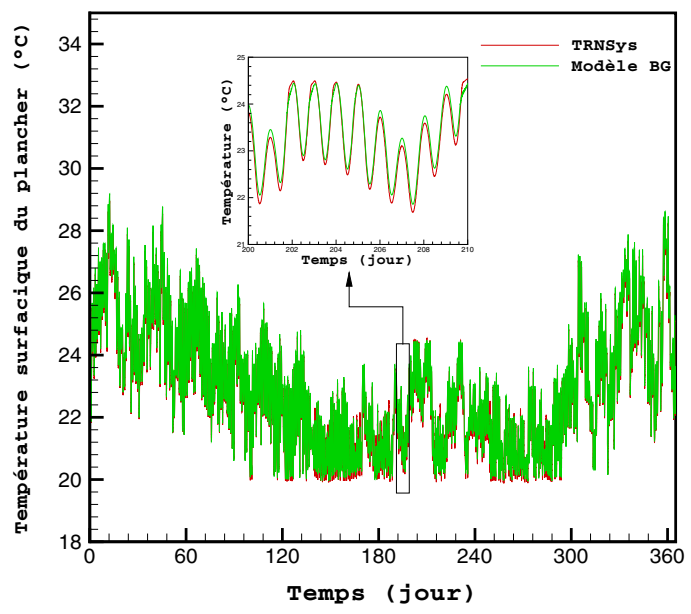

(a)

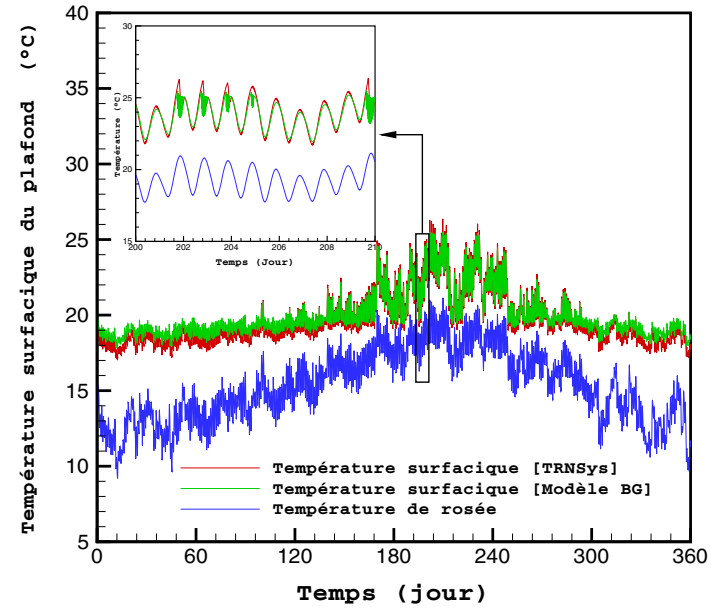

(b)

Fig. 9. Évolution des températures surfaciques (zone 1). (a) Plancher chauffant; (b) plafond refroidissant.

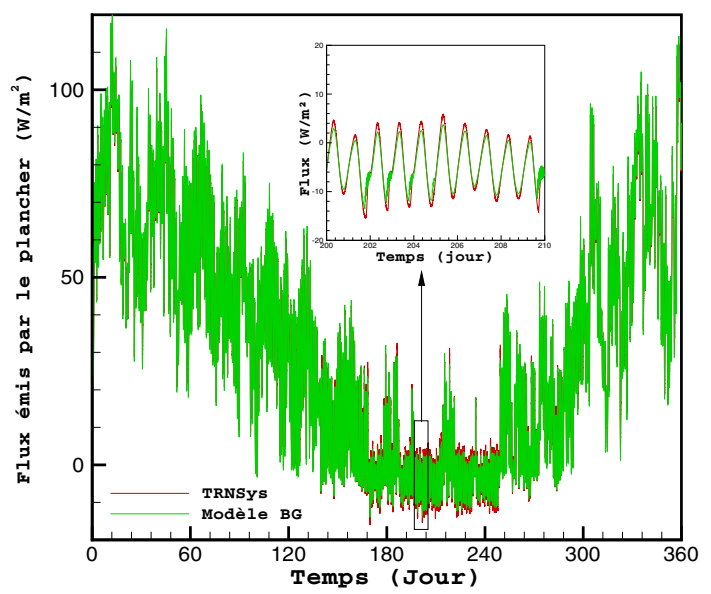

(a)

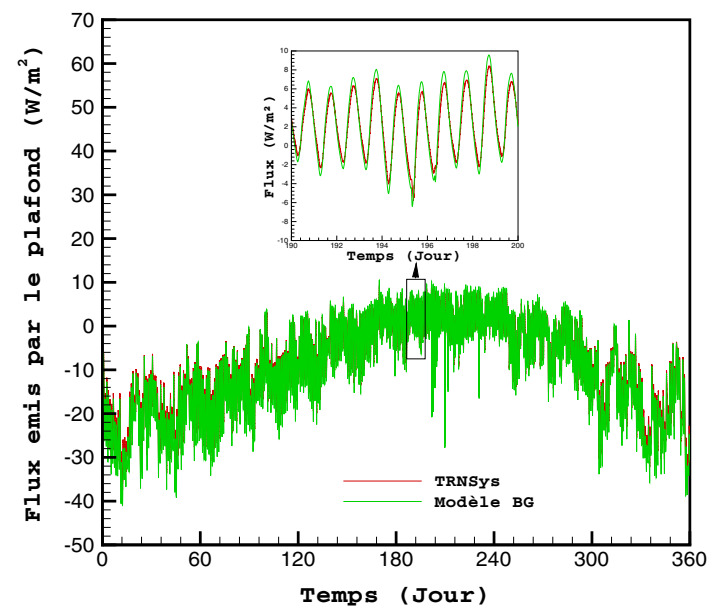

(b)

Fig. 10. Évolution des flux de chaleur (zone 1). (a) Plancher chauffant; (b) plafond refroidissant.

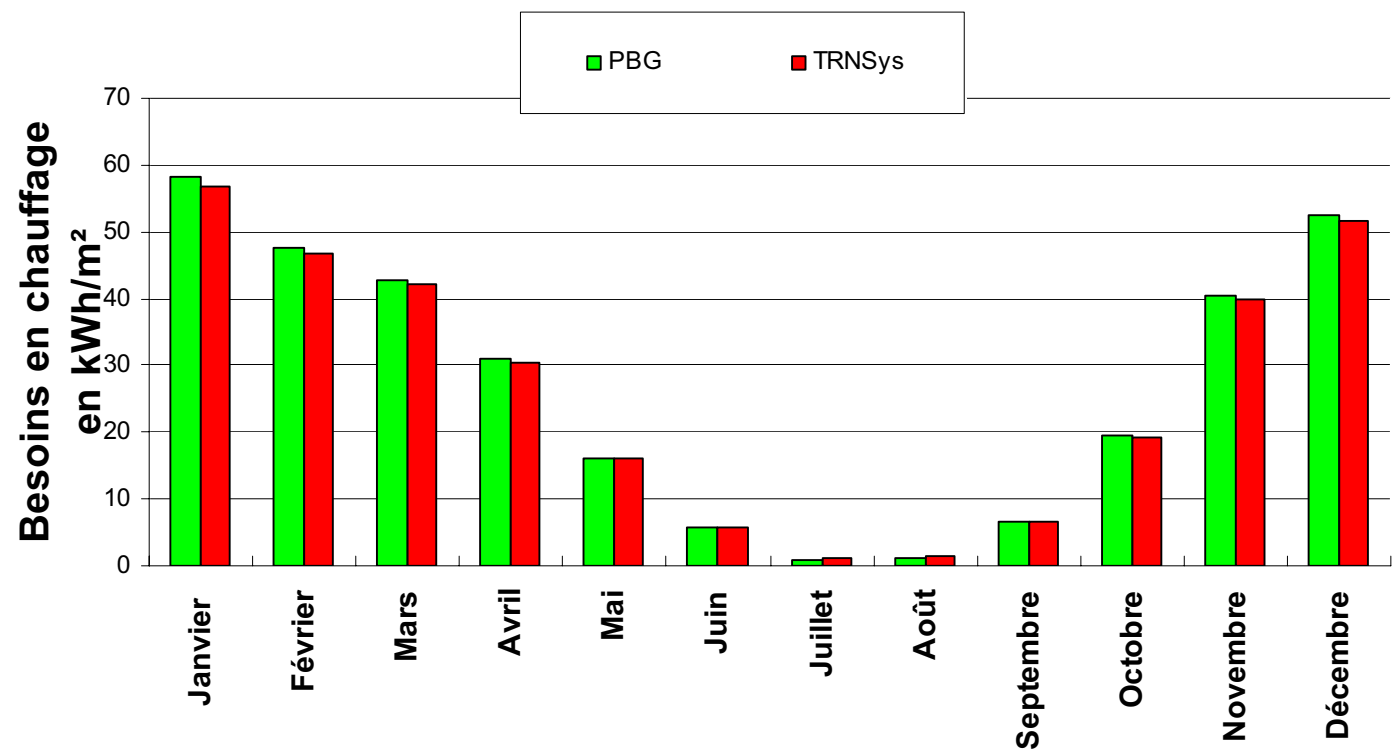

Fig. 11. Besoins mensuels en chauffage. 


\section{Conclusion}

Une étude de modélisation du comportement thermique d'un bâtiment, intégrant à la fois un plancher chauffant et un plafond rafraîchissant, a été réalisée. La méthodologie Bond Graph, jusqu'ici peu employée dans la modélisation des systèmes thermiques, a été utilisée. Les simulations effectuées ont permis de valider le modèle global du bâtiment étudié. Cet outil se veut pérenne tant pour une exploitation en recherche que pour une utilisation en ingénierie.

En utilisant un système de régulation de la température de l'air, il s'est avéré que la zone 1 satisfait les critères du confort thermique. La température radiante est comprise entre $19{ }^{\circ} \mathrm{C}$ et $27^{\circ} \mathrm{C}$. Étant sans chauffage en hiver et sans rafraîchissement en été, la température intérieure de la zone 2 est relativement basse en hiver.

L'intérêt d'une telle technique par rapport aux systèmes classiques de chauffage et de rafraîchissement est que celui-ci est caractérisé par le fait que l'apport de chaleur et de froid est réparti uniformément dans la pièce, ce qui procure un confort thermique supérieur aux autres types de chauffage et de rafraîchissement. Une autre caractéristique est que le confort est renforcé par l'absence de courant d'air et d'encombrement.

Cependant, il serait intéressant de comparer les consommations, en énergie primaire, de ce système combiné aux systèmes conventionnels.

\section{Références}

[1] B. Olesen, Radiant floor cooling systems, ASHRAE J. (2008) 16-22

[2] M. Amir, M. Lacroix, N. Galanis, Comportement thermique de dalles chauffantes électriques pour le stockage quotidien, Int. J. Therm. Sci. 38 (1999) 121-131

[3] S. Larsen, C. Filippin, G. Lesino, Transient simulation of a storage floor with a heating/cooling parallel pipe system, Build. Simul.: An Int. J. 3 (2010) 105-115

[4] A. Mokhtari, H. Kazeoui, Y. Boukezzi, G. Achard, Utilisation d'un circuit hydraulique dans un plancher pour le chauffage et le rafraîchissement des locaux, Revue des Énergies Renouvelables 1 (1998) 17-27

[5] N.F. Diaz, Modeling of a hydronic ceiling system and its environment as energetic auditing tool, J. Appl. Energ. 88 (2011) 636-649

[6] A. Keblawi, N. Ghaddar, K. Ghali, Model-based optimal supervisory control of chilled ceiling displacement ventilation system, J. Energ. Build. 43 (2011) 1359-1370
[7] X. Hao, G. Zhang, Y. Chen, S. Zou, D.J. Moschandreas, A combined system of chilled ceiling, displacement entilation and desiccant deshumidification, J. Build. Environ. 42 (2007) 3298-3308

[8] N. Paynter, Analysis and design of engineering systems, MIT Press, 1961

[9] B. Ould Bouamama, G. Dauphin-Tanguy, Modelisation par Bond Graphs : Élement de base pour l'énergétique, Techniques de l'ingénieur (2006) BE8280

[10] B. Ould Bouamama, G. Dauphin-Tanguy, Modelisation par Bond Graphs : Application aux systèmes énergétiques, Techniques de l'ingénieur (2006) BE8281

[11] J. Thoma, B. Ould Bouamama, Modeling and simulation in thermal and chemical engineering, Bond Graph approach, Springer-Verlag, 2000

[12] F.E. Cellier, A. Nebot, Bond Graphs modelling of heat and humidity budgets of Biosphere 2, Environmental modelling and software (2006) 1598-1606

[13] W. Borutzky, Bond Graph methodology: Development and analysis of multidisciplinary dynamic system models, Springer-Verlag, London, 2010

[14] D. Karnopp, S. Azarbaijani, Pseudo Bond Graph for generalized compartmental models in engineering and physiology, J. Frank. Inst. 312 (1981) 95-08

[15] D. Karnopp, State variables and pseudo Bond Graphs for compressible thermofluid systems, J. Dyn. Syst., Meas. Control 101 (1979) 201-204

[16] A. Merabtine, S. Mokraoui, R. Benelmir, D. Descieux, A Comparative modeling of an experimental single-zone building with Bond Graphs and TRNSYS, Int. J. Energy, Environment, and Economics 20 (2012) 39-51

[17] A. Merabtine, S. Mokraoui, R. Benelmir, N. Laraqi, Bond Graph approach for modeling and analysis of transient heat conduction in the prospect of energy building application, Int. J. Energy, Environment, and Economics 20 (2012) 53-73

[18] Réglementation Thermique 2005, Guide réglementaire 2005, CSTB

[19] A. Baïri, N. Laraqi, J.M. García de María, Numerical and experimental study of natural convection in tilted parallelepipedic cavities for large Rayleigh numbers, Exp. Therm. Fluid Sci. 31 (2007) 309-324

[20] A. Baïri, N. Laraqi, J.M. García de María, Importance of radiative heat exchange in $2 \mathrm{D}$ closed diode cavities applied to solar collectors and building, Int. J. Sustainable Energy 24 (2005) 33-44

[21] D. Medjelekh, S. Abdou, M. El Ganaoui, Impact of the Thermal Inertia of Material on the Hygrothermal Comfort of Building, Int. Rev. Chem. Eng. 2 (2010) 391397 